\title{
Hypo-exponential distributions and compound Poisson processes with alternating parameters
}

\author{
Nikita Ratanov \\ Universidad del Rosario, Cl.12c, No. 4-69, Bogotá, Colombia
}

\section{A R T I C L E I N F O}

\section{Article history:}

Received 1 June 2015

Received in revised form 7 August 2015

Accepted 7 August 2015

Available online 14 August 2015

\section{MSC:}

primary 60J27

secondary $60 J 75$

60K99

Keywords:

Poisson process

Markov-modulated Poisson process

Erlang distribution

Hypo-exponential distribution

Hyper-exponential distribution

\begin{abstract}
A B S T R A C T
Point processes with alternating arrival rates arise in various applications, including financial modelling. We obtain explicit expressions for the distributions of these processes, i.e. for the sums $\sum_{m=1}^{n} X^{(m)}$ and $\sum_{m=1}^{n}(-1)^{m} X^{(m)}$, where $X^{(m)}$ are independent exponentially distributed random variables with alternating parameters.

The distribution of the compound Poisson process with Markov modulation and with exponentially distributed jumps is also studied.
\end{abstract}

(C) 2015 Elsevier B.V. All rights reserved.

\section{Introduction}

Sums of independent and identically (exponentially) distributed random variables having the Erlang distribution are well studied. Random motions with constant velocities alternating at Erlang-distributed times have been studied in Di Crescenzo (2001).

If all terms have different exponential distributions, the distribution is called a generalised Erlang (or hypo-exponential), and this case is also well known, see e.g. Ross (2007).

However, the case of a sum of the exponentially distributed terms with alternating parameters is not studied. This case is important for applications, notably for financial modelling, see e.g. Ratanov (2007) and Kolesnik and Ratanov (2013). The complete and arbitrage-free market models based on the Poisson (Cox) processes with alternating random switching intensities have been studied by Ratanov (2014). The financial market models based on the telegraph processes with random jumps (e.g. with the exponentially distributed jumps) have been studied before by López et al. (2012) and Ratanov (2015), see also Ratanov (2013). Some generalisation of the hypo-exponential distribution recently appears for reliability modelling, see Saboor et al. (2015).

This paper concerns the two main different cases: the hypo-exponential distribution with the terms of the same sign and the hypo-exponential distribution with alternating signs of summands. The distributions of terms are assumed to be alternating. The analysis is based on convolutions of the standard Erlang distributions. These convolutions are expressed explicitly by the confluent hypergeometric Kummer functions (if the jumps have the same signs) and by the Bessel polynomials (if the jumps have alternating signs).

E-mail address: nikita.ratanov@urosario.edu.co. 
The compound Poisson processes (with Poisson subordinator and with i.i.d. jumps) are recently studied by Di Crescenzo et al. (2015). The present paper generalises some of the results Di Crescenzo et al. (2015) to the case with alternating parameters.

Then, we study the marginal distributions of a Markov modulated compound Poisson process with alternating parameters. The similar problems on the Markov modulated compound Poisson process are studied by Xu et al. (2015) by applying the double Laplace transform with respect to the level and time of the process. See there some numerical results and plots of the marginal distributions.

In the present paper we study the distributions of the alternating sums of the exponentially distributed independent random variables by direct methods. As a by-product we collect some useful explicit formulae.

In Section 2 we get hypo-exponential distributions with alternating parameters. The cases with the same and with alternating signs are discussed separately. In Sections 3 and 4 we study various forms of compound Poisson processes with alternating states by applying the results of Section 2 .

\section{Hypo-exponential distributions with alternating parameters}

Let $X^{(n)}, \quad n \geq 1$, be independent random variables, which are distributed with alternating cumulative distribution functions $G_{0}$ and $G_{1}$. Consider the jump process

$$
X^{(+, n)}=X^{(1)}+X^{(2)}+\cdots+X^{(n)}, \quad n \geq 1 .
$$

Assume that $X^{(+, 0)}=0$. Note that the cumulative distribution function of the sum $X^{(+, n)}, n \geq 1$, is given by the $n$-fold convolution of alternating $G_{0}$ and $G_{1}$ (beginning with $\left.G_{k}\right), G_{k}^{(*, n)}(x)=\underbrace{G_{k} * G_{1-k} * G_{k} * \cdots * G_{\sigma_{n, k}}}_{n}$, and $g_{k}^{(*, n)}=g\left(\cdot ; n ; G_{k}\right.$,

$G_{1-k}$ ) are the corresponding density functions, $k \in\{0,1\}$. Here $\sigma_{n, k}=k$, if $n$ is odd, and $\sigma_{n, k}=1-k$, if $n$ is even.

By symmetry, $g\left(x ; 2 n ; G_{0}, G_{1}\right) \equiv g\left(x ; 2 n ; G_{1}, G_{0}\right)$.

In this section we provide explicit formulae for the distribution of the jump process $X^{(+, n)}$, assuming the alternating exponential distributions of jumps $X^{(n)}$. Consider first the process with positive jumps.

\subsection{Positive exponential jumps}

If $X^{(n)}, n \geq 1$, are identically and exponentially distributed, $G_{0}(x) \equiv G_{1}(x)=\left(1-\mathrm{e}^{-a x}\right) \mathbb{1}_{\{x>0\}}$, the variable $X^{(+, n)}$ has the standard Erlang- $n$ distribution with the density function

$$
f(x ; n ; a)=\frac{a^{n} x^{n-1}}{(n-1) !} \mathrm{e}^{-a x} \mathbb{1}_{\{x>0\}} .
$$

If the alternating exponential distributions $G_{0}$ and $G_{1}$ are different, $G_{0} \neq G_{1}$, we say, that the distribution of $X^{(+, n)}$ is the generalised Erlang (or hypo-exponential) distribution with alternating intensities.

Proposition 2.1. Let jumps $X^{(n)}$ be positive and exponentially distributed with the alternating cumulative distribution functions

$$
G_{0}(x)=\left(1-\exp \left(-a_{0} x\right)\right) \mathbb{1}_{\{x>0\}} \quad \text { and } \quad G_{1}(x)=\left(1-\exp \left(-a_{1} x\right)\right) \mathbb{1}_{\{x>0\}}, \quad a_{0}, a_{1}>0 .
$$

The density functions $g\left(\cdot ; n ; G_{0}, G_{1}\right)$ of $X^{(+, n)}$ are given by

$$
g\left(x ; n ; G_{0}, G_{1}\right)=a_{0}^{(\times, n)} \cdot \frac{x^{n-1}}{(n-1) !} \exp \left(-a_{0} x\right) \Phi\left([n / 2] ; n ;\left(a_{0}-a_{1}\right) x\right) \mathbb{1}_{\{x>0\}}, \quad n \geq 1 .
$$

Here

$$
a_{0}^{(\times, n)}:=\underbrace{a_{0} \cdot a_{1} \cdot a_{0} \cdot \ldots \cdot a_{\sigma_{n, 0}}}_{n}= \begin{cases}a_{0}^{n / 2} a_{1}^{n / 2}, & \text { if } n \text { is even } \\ a_{0}^{(n+1) / 2} a_{1}^{(n-1) / 2}, & \text { if } n \text { is odd, }\end{cases}
$$

$[n / 2]$ is the integer part of $n / 2$ and $\Phi(\cdot ; \cdot ; z)$ is the confluent hypergeometric Kummer function.

Note that the identity $g\left(x ; 2 n ; G_{0}, G_{1}\right) \equiv g\left(x ; 2 n ; G_{1}, G_{0}\right)$ (which is valid by symmetry) follows from (2.3) by formula (9.212.1), Gradshteyn and Ryzhik (1980). Moreover, by (2.3) with $n=1$ we have, see (9.211.2), Gradshteyn and Ryzhik (1980),

$$
g\left(x ; 2 ; G_{0}, G_{1}\right) \equiv g\left(x ; 2 ; G_{1}, G_{0}\right)=\frac{a_{0} a_{1}}{a_{0}-a_{1}}\left(\mathrm{e}^{-a_{1} x}-\mathrm{e}^{-a_{0} x}\right) \mathbb{1}_{\{x>0\}},
$$

which is the well-known two-phase hypo-exponential distribution, see e.g. Ross (2007). If $a_{0}=a_{1}$, formula (2.3) coincides with the standard Erlang- $n$ distribution, (2.2). 
Proof. We prove (2.3) with an even number of jumps, in the case of an odd $n$ the proof is similar.

The density function $g\left(\cdot ; 2 n ; G_{0}, G_{1}\right)$ is represented by convolution of two Erlang- $n$ densities, $(2.2)$,

$$
\begin{aligned}
g\left(x ; 2 n ; G_{0}, G_{1}\right) & =g\left(x ; 2 n ; G_{1}, G_{0}\right)=\int_{0}^{x} f\left(x-y ; n ; a_{0}\right) f\left(y ; n ; a_{1}\right) \mathrm{d} y \\
& =\frac{a_{0}^{n} a_{1}^{n}}{[(n-1) !]^{2}} \mathrm{e}^{-a_{0} x} \int_{0}^{x}(x-y)^{n-1} y^{n-1} \mathrm{e}^{\left(a_{0}-a_{1}\right) y} \mathrm{~d} y, \quad x>0 .
\end{aligned}
$$

By applying (3.383.1), Gradshteyn and Ryzhik (1980) we obtain formula (2.3) for the sum with an even number of terms.

Remark 2.1. Let $G_{0}(x)=\left(1-\exp \left(-a_{0} x\right)\right) \mathbb{1}_{\{x>0\}}$ and $G_{1}(x)=\left(1-\exp \left(-a_{1} x\right)\right) \mathbb{1}_{\{x>0\}}, a_{0}, a_{1}>0$. Note that conditioning on the first arrival we have

$$
g\left(x ; n ; G_{0}, G_{1}\right)=\int_{0}^{x} a_{0} \mathrm{e}^{-a_{0} y} g\left(x-y ; n-1 ; G_{1}, G_{0}\right) \mathrm{d} y, \quad n \geq 1 .
$$

The set of the integral equations (2.5) is equivalent to

$$
\hat{g}\left(\xi ; n ; G_{0}, G_{1}\right)=\frac{a_{0}}{\xi+a_{0}} \hat{g}\left(\xi ; n-1 ; G_{1}, G_{0}\right), \quad n \geq 1,
$$

where $\hat{g}(\xi)=\int_{0}^{\infty} \mathrm{e}^{-\xi x} g(x) \mathrm{d} x$ is the Laplace transform.

Since $X^{(+, 0)}=0$, system (2.6) is supplied with the initial equation $\hat{g}\left(\xi ; 0 ; G_{0}, G_{1}\right)=1$. One can easily obtain the solution of $(2.6)$ :

$$
\hat{g}\left(\xi ; n ; G_{0}, G_{1}\right)=\frac{a_{0}^{(\times, n)}}{\left(\xi+a_{0}\right)^{(\times, n)}}, \quad n \geq 1 .
$$

By applying the inverse Laplace transform to (2.7) one can get the different proof of (2.3).

\subsection{Exponential jumps with alternating signs}

Bearing in mind financial applications assume jumps $X^{(n)}$ in (2.1) to be of alternating signs. Let

$$
G_{0}(x)=\left\{\begin{array}{ll}
\exp \left(a_{0} x\right), & \text { if } x<0 \\
1, & \text { if } x>0
\end{array}, \quad G_{1}(x)=\left(1-\exp \left(-a_{1} x\right)\right) \mathbb{1}_{\{x>0\}}\right.
$$

be their cumulative distribution functions.

We express the density functions of $X^{(+, n)}$ by the Bessel polynomial

$$
y_{n-1}(z):=\sum_{m=0}^{n-1} \frac{(n+m-1) !}{m !(n-m-1) !}(z / 2)^{m}, \quad n \geq 1,
$$

(see Krall and Frink (1948)), and its derivative $y_{n-1}^{\prime}(z)=\frac{1}{2} \sum_{m=1}^{n-1} \frac{(n+m-1) !}{(m-1) !(n-m-1) !}(z / 2)^{m-1}$.

Proposition 2.2. The density functions $g\left(x ; n ; G_{0}, G_{1}\right)$ are given by

$$
\begin{aligned}
& g\left(x ; 2 n ; G_{0}, G_{1}\right)=h_{n}(x) y_{n-1}(\alpha(x)), \\
& g\left(x ; 2 n+1 ; G_{0}, G_{1}\right)=\frac{a_{0}}{a_{0}+a_{1}} h_{n}(x) \begin{cases}y_{n-1}(\alpha(x))+\frac{\alpha(x)}{n} y_{n-1}^{\prime}(\alpha(x)), & x>0, \\
\left(1+\frac{2}{n \alpha(x)}\right) y_{n-1}(\alpha(x))+\frac{\alpha(x)}{n} y_{n-1}^{\prime}(\alpha(x)), & x<0,\end{cases} \\
& g\left(x ; 2 n+1 ; G_{1}, G_{0}\right)=\frac{a_{1}}{a_{0}+a_{1}} h_{n}(x) \begin{cases}\left(1+\frac{2}{n \alpha(x)}\right) y_{n-1}(\alpha(x))+\frac{\alpha(x)}{n} y_{n-1}^{\prime}(\alpha(x)), & x>0, \\
y_{n-1}(\alpha(x))+\frac{\alpha(x)}{n} y_{n-1}^{\prime}(\alpha(x)), & x<0,\end{cases}
\end{aligned}
$$

$n \geq 1$. Here $\alpha(x)=\frac{2}{\left(a_{0}+a_{1}\right)|x|}, h_{n}(x)=\frac{a_{0}^{n} a_{1}^{n}}{\left(a_{0}+a_{1}\right)^{n}} \frac{|x|^{n-1} \theta(x)}{(n-1) !}$ and $\theta(x)=\left\{\begin{array}{ll}\mathrm{e}^{-a_{1} x}, & \text { if } x>0 \\ \mathrm{e}^{a_{0} x}, & \text { if } x<0\end{array}\right.$.

Note that the density functions (2.9) with $n=1$ correspond to the asymmetric Laplace distribution, Kotz et al. (2001),

$$
g\left(x ; 2 ; G_{0}, G_{1}\right)=\frac{a_{0} a_{1}}{a_{0}+a_{1}} \theta(x), \quad-\infty<x<\infty .
$$


Proof. Similarly to the proof of the previous proposition, see (2.4), we obtain formulae (2.9)-(2.10) by the convolution of two Erlang distributions. For instance, in the case of even number of jumps,

$$
g\left(x ; 2 n ; G_{0}, G_{1}\right)=g\left(x ; 2 n ; G_{1}, G_{0}\right)= \begin{cases}\int_{0}^{\infty} f\left(y ; n ; a_{0}\right) f\left(x+y ; n ; a_{1}\right) \mathrm{d} y, & \text { if } x>0, \\ \int_{0}^{\infty} f\left(y ; n ; a_{1}\right) f\left(-x+y ; n ; a_{0}\right) \mathrm{d} y, & \text { if } x<0,\end{cases}
$$

where $f$ is the Erlang density (2.2).

If $x>0$, we have

$$
\begin{aligned}
g\left(x ; 2 n ; G_{0}, G_{1}\right)=g\left(x ; 2 n ; G_{1}, G_{0}\right) & =\frac{a_{0}^{n} a_{1}^{n}}{[(n-1) !]^{2}} \mathrm{e}^{-a_{1} x} \int_{0}^{\infty}(x+y)^{n-1} y^{n-1} \mathrm{e}^{-\left(a_{0}+a_{1}\right) y} \mathrm{~d} y \\
& =\frac{a_{0}^{n} a_{1}^{n}}{[(n-1) !]^{2}} x^{2 n-1} \mathrm{e}^{-a_{1} x} \sum_{m=0}^{n-1} \frac{(n-1) !}{m !(n-m-1) !} \int_{0}^{\infty} y^{n+m-1} \mathrm{e}^{-\left(a_{0}+a_{1}\right) x y} \mathrm{~d} y \\
& =\frac{a_{0}^{n} a_{1}^{n}}{(n-1) !} x^{2 n-1} \mathrm{e}^{-a_{1} x} \sum_{m=0}^{n-1} \frac{(n+m-1) !}{m !(n-m-1) !}\left[\left(a_{0}+a_{1}\right) x\right]^{-n-m},
\end{aligned}
$$

which gives (2.9) (for $x>0$ ). In the case of $x<0$ we proceed similarly.

Likewise, we obtain for $n \geq 1$

$$
g\left(x ; 2 n+1 ; G_{0}, G_{1}\right)= \begin{cases}\int_{0}^{\infty} f\left(y ; n+1 ; a_{0}\right) f\left(x+y ; n ; a_{1}\right) \mathrm{d} y, & \text { if } x>0, \\ \int_{0}^{\infty} f\left(y ; n ; a_{1}\right) f\left(-x+y ; n+1 ; a_{0}\right) \mathrm{d} y, & \text { if } x<0,\end{cases}
$$

and

$$
g\left(x ; 2 n+1 ; G_{1}, G_{0}\right)= \begin{cases}\int_{0}^{\infty} f\left(y ; n ; a_{0}\right) f\left(x+y ; n+1 ; a_{1}\right) \mathrm{d} y, & \text { if } x>0, \\ \int_{0}^{\infty} f\left(y ; n+1 ; a_{1}\right) f\left(-x+y ; n ; a_{0}\right) \mathrm{d} y, & \text { if } x<0 .\end{cases}
$$

We complete the proof by applying to formulae (2.13)-(2.14) the manipulations similar to the case of even number of jumps.

Remark 2.2. Note, that if the independent jumps $X^{(n)}$ are identically distributed with the asymmetric Laplace distribution (the density function is $g(x)=\frac{a_{0} a_{1}}{a_{0}+a_{1}} \theta(x),-\infty<x<\infty, a_{0}, a_{1}>0$, see (2.12)), then the density functions of $X^{(+, n)}$ are given by (2.9).

Example 2.1. Let the independent jumps $\left\{X^{(n)}\right\}_{n \geq 1}$ be exponentially distributed with common parameter, $\operatorname{Exp}(a)$, and $N=N(t)$ be the homogeneous Poisson process with parameter $\lambda$. Consider the Cramér-Lundberg risk process (the compound Poisson process with drift)

$$
Y(t)=c t-X^{(+, N(t))} .
$$

Let $\tau_{n}, n \geq 1$, be arrival times. The distribution of $Y\left(\tau_{n}\right)$ is given by (2.9) with parameters $a$ and $\lambda / c$ instead of $a_{0}$ and $a_{1}$.

The similar approach can be applied to a jump-telegraph process driven by $N(t)$, which is supplied with exponential jumps $X^{(n)}$.

\section{Compound Poisson processes with alternating states}

Let $\sigma=\sigma(t) \in\{0,1\}, t \geq 0$, be a two-state self-exciting right-continuous Markov process independent of $\left\{X^{(n)}\right\}_{n \geq 1}$ with the alternating intensities $\lambda_{0}, \lambda_{1}>0$ :

$$
\mathbb{P}\{\sigma(t+\mathrm{d} t) \neq \sigma(t) \mid \sigma(t)\}=\lambda_{\sigma(t)} \mathrm{d} t+o(\mathrm{~d} t), \quad \mathrm{d} t \rightarrow 0 .
$$

The time intervals $\left\{\tau^{(n)}\right\}_{n \geq 1}$ between the consequent switchings of $\sigma$ are independent exponentially distributed random variables with alternating intensities $\lambda_{0}$ and $\lambda_{1}$. By summing up $\tau^{(n)}, n \geq 1$, one can obtain the flow of switching instants

$$
\tau^{(+, n)}:=\tau^{(1)}+\tau^{(2)}+\cdots+\tau^{(n)} ; \quad \tau^{(+, 0)}:=0 .
$$

Let $N(t):=\max \left\{n: \tau^{(+, n)} \leq t\right\}, t \geq 0$, be the counting process, which can be considered as the Cox process with the instantaneous random switching intensities $\lambda(t)=\lambda_{\sigma(t)}$. Notice that the underlying Markov process $\sigma$ (state variable) is 
given by

$$
\sigma(t)=1-\sigma_{N(t), k}, \quad t \geq 0, k \in\{0,1\} .
$$

The distributions of the sums $\tau^{(+, n)}, n \geq 1$, defined by (3.2), are given by Proposition 2.1 with $\lambda_{0}, \lambda_{1}$ instead of $a_{0}, a_{1}$.

Denote the density function of $\tau^{(+, n)}$ by $f_{0}(t ; n)=f_{0}\left(t ; n ; \lambda_{0}, \lambda_{1}\right)$, (if $\tau^{(1)} \sim \operatorname{Exp}\left(\lambda_{0}\right)$ ). If the initial state is 1 , function $f_{1}(t ; n)=f_{1}\left(t ; n ; \lambda_{0}, \lambda_{1}\right)=f_{0}\left(t ; n ; \lambda_{1}, \lambda_{0}\right)$. Functions $f_{k}(t ; n), k \in\{0,1\}, n \geq 1$, were obtained in Proposition 2.1, see (2.3).

Let $\pi_{k}(t ; n)=\pi_{k}\left(t ; n ; \lambda_{0}, \lambda_{1}\right):=\mathbb{P}\{N(t)=n \mid \sigma(0)=k\}, n \geq 0$, be the probability mass function of $N(t)$.

Conditioning on the first switching of $\sigma(t)$ similarly to (2.5) we obtain two systems of integral equations: for $n \geq 1$

$$
\begin{aligned}
& \pi_{k}(t ; n)=\int_{0}^{t} \lambda_{k} \mathrm{e}^{-\lambda_{k} u} \pi_{1-k}(t-u ; n-1) \mathrm{d} u, \\
& f_{k}(t ; n)=\int_{0}^{t} \lambda_{k} \mathrm{e}^{-\lambda_{k} u} f_{1-k}(t-u ; n-1) \mathrm{d} u .
\end{aligned}
$$

Eqs. (3.3) and (3.4) are supplied with the initial functions:

$$
\pi_{k}(t ; 0)=\mathbb{P}\{N(t)=0 \mid \sigma(0)=k\}=\mathrm{e}^{-\lambda_{k} t}, \quad t \geq 0, \quad \text { and } f_{k}(t ; 0)=\delta(t), k \in\{0,1\},
$$

where $\delta=\delta(t)$ is Dirac's $\delta$-function. By (3.3)-(3.4) we have $f_{k}(t ; 1)=\lambda_{k} \mathrm{e}^{-\lambda_{k} t}=\lambda_{k} \pi_{k}(t ; 0)$ and then,

$$
\pi_{k}\left(t ; n ; \lambda_{0}, \lambda_{1}\right)=\frac{f_{k}\left(t ; n+1 ; \lambda_{0}, \lambda_{1}\right)}{\lambda_{\sigma_{n+1, k}}}, \quad t \geq 0, n \geq 1, k \in\{0,1\} .
$$

From (2.3) and Eq. (3.5) (with $\lambda_{0}, \lambda_{1}$ instead of $a_{0}, a_{1}$ ) one can obtain

$$
\pi_{k}(t ; n)=\frac{\lambda_{k}^{(\times, n)} t^{n}}{n !} \mathrm{e}^{-\lambda_{k} t} \Phi\left(\left[\frac{n+1}{2}\right], n+1 ;\left(\lambda_{k}-\lambda_{1-k}\right) t\right), \quad t \geq 0, n \geq 1, k \in\{0,1\} .
$$

We study the compound Poisson process $J(t)$ with jumps $X^{(n)}$ occurring at times $\tau^{(+, n)}$,

$$
J(t):=X^{(+, N(t))},
$$

where sums $X^{(+, n)}$ are defined by (2.1).

If the initial state is $k=\sigma(0)$, the distribution function $\Phi_{k}(x, t)$ of $J(t)$ is given by

$$
\Phi_{k}(x, t)=\mathbb{P}\{(t) \leq x \mid \sigma(0)=k\}=\mathrm{e}^{-\lambda_{k} t} \mathbb{1}_{\{x \geq 0\}}+\sum_{n=1}^{\infty} \pi_{k}\left(t ; n ; \lambda_{0}, \lambda_{1}\right) G_{k}^{(*, n)}(x),
$$

where $\pi_{k}(t ; n)$ is given by (3.6) and $G_{k}^{(*, n)}(x)$ is the alternating $n$-fold convolution of the distributions of jumps, Section 2 .

The corresponding density function is

$$
\varphi_{k}(x, t)=\mathrm{e}^{-\lambda_{k} t} \delta(x)+\sum_{n=1}^{\infty} \pi_{k}\left(t ; n ; \lambda_{0}, \lambda_{1}\right) g\left(x ; n ; G_{k}, G_{1-k}\right) .
$$

In the case of positive exponentially distributed jumps we have

$$
\varphi_{k}(x, t)=\mathrm{e}^{-\lambda_{k} t} \delta(x)+\sum_{n=1}^{\infty} \lambda_{\sigma_{n+1}, k}^{-1} f_{k}\left(t ; n+1 ; \lambda_{0}, \lambda_{1}\right) f_{k}\left(x ; n ; a_{0}, a_{1}\right),
$$

where $f_{k}(t ; \cdot ; \cdot, \cdot)$ are given by $(2.3)$.

Formulae (3.8)-(3.10) generalise the results of Di Crescenzo et al. (2015, Section 4).

Let $T=T^{\mu}$ be exponentially distributed, $\operatorname{Exp}(\mu)$, independent of $\sigma$ and $X^{(n)}, n \geq 1$, random variable. Consider the compound Poisson process $J(t)$ stopped at time $T$. The density function of the random variable $J(T)$ is given by $\mu \hat{\varphi}_{k}(x ; \mu)$, where $\hat{\varphi}_{k}(x ; \mu)=\int_{0}^{\infty} \mathrm{e}^{-\mu t} \varphi_{k}(x, t) \mathrm{d} t$. The Laplace transform $\hat{\varphi}(x ; \mu)$ takes the form:

$$
\hat{\varphi}_{k}(x ; \mu)=\frac{\delta(x)}{\mu+\lambda_{k}}+\sum_{n=1}^{\infty} \hat{\pi}_{k}(\mu ; n) g\left(x ; n ; G_{0}, G_{1}\right),
$$

where $\hat{\pi}_{k}(\mu ; n):=\int_{0}^{\infty} \mathrm{e}^{-\mu t} \pi_{k}(t ; n) \mathrm{d} t$. Due to (2.7) and (3.5)

$$
\hat{\varphi}_{k}(x ; \mu)=\frac{\delta(x)}{\mu+\lambda_{k}}+\sum_{n=1}^{\infty} \frac{\lambda_{k}^{(\times, n)}}{\left(\mu+\lambda_{k}\right)^{(\times, n+1)}} g\left(x ; n ; G_{0}, G_{1}\right) .
$$


Remark 3.1. In the case of identical exponential distributions of jumps (Proposition 2.1 with $a_{0}=a_{1}=a$ ) formula ( 3.11 ) can be obtained in the simple closed form. In this case $g\left(x ; n ; G_{0}, G_{1}\right)=f(x ; n ; a)=\frac{a^{n} x^{n-1}}{(n-1) !} \mathrm{e}^{-a x} \mathbb{1}_{\{x>0\}}$ is the standard Erlang distribution. We have

$$
\hat{\varphi}_{k}(x ; \mu)=\frac{\delta(x)}{\mu+\lambda_{k}}+\frac{\mathrm{e}^{-a x}}{x} \sum_{n=1}^{\infty} \frac{(a x)^{n}}{(n-1) !} \frac{\lambda_{k}^{(\times, n)}}{\left(\mu+\lambda_{k}\right)^{(\times, n+1)}} \mathbb{1}_{\{x>0\}}
$$

and after easy algebra we obtain the densities of the random variable $J(T)$,

$$
\begin{aligned}
& \mu \hat{\varphi}_{0}(x ; \mu)=\frac{\mu}{\mu+\lambda_{0}}\left[\delta(x)+a \beta_{0} \exp (-a x)\left(\beta_{0} \cosh \left(\beta_{0} \beta_{1} a x\right)+\beta_{1} \sinh \left(\beta_{0} \beta_{1} a x\right)\right)\right], \\
& \mu \hat{\varphi}_{1}(x ; \mu)=\frac{\mu}{\mu+\lambda_{1}}\left[\delta(x)+a \beta_{1} \exp (-a x)\left(\beta_{1} \cosh \left(\beta_{0} \beta_{1} a x\right)+\beta_{0} \sinh \left(\beta_{0} \beta_{1} a x\right)\right)\right],
\end{aligned}
$$

where

$$
\beta_{0}=\sqrt{\frac{\lambda_{0}}{\mu+\lambda_{1}}}, \quad \beta_{1}=\sqrt{\frac{\lambda_{1}}{\mu+\lambda_{0}}} .
$$

\section{Markov-modulated compound Poisson processes}

Let $\varepsilon=\varepsilon(t), t \geq 0$, be the right-continuous 2-state Markov chain with the infinitesimal generator

$$
\mathscr{M}=\left(\begin{array}{cc}
-\mu_{0} & \mu_{0} \\
\mu_{1} & -\mu_{1}
\end{array}\right) \text {. }
$$

Denote by $\varepsilon_{i}$ the process $\varepsilon=\varepsilon(t), t \geq 0$, under the given initial state $i, \varepsilon_{i}(0)=i$.

Consider the doubly stochastic counting Poisson process $N=N(t)$ with instantaneous arrival rates, $\lambda_{\varepsilon(t)}, \lambda_{0}, \lambda_{1}>0$, that are controlled by the underlying process $\varepsilon$.

More precisely, in contrast with Section 3, denote by $\left\{\tau^{(+, n)}\right\}_{n \geq 1}$ the flow of instants with independent interarrival intervals $\tau^{(n)}$, assuming that when the Markov chain $\varepsilon(t)$ is in state $j, j \in\{0,1\}$, the arrivals occur at rate $\lambda_{j}$.

Process $N=N(t)=N_{0}(t)+N_{1}(t)$ counts the number of arrivals in $[0, t]$, where $N_{0}(t)$ and $N_{1}(t)$,

$$
N_{0}(t)=\sum_{n=1}^{\infty} \mathbb{1}_{\left\{\tau^{(+, n)} \leq t, \varepsilon\left(\tau^{(+, n)}\right)=0\right\}}, \quad N_{1}(t)=\sum_{n=1}^{\infty} \mathbb{1}_{\left\{\tau^{(+, n)} \leq t, \varepsilon\left(\tau^{(+, n)}\right)=1\right\}},
$$

count separately the arrivals that occur at the states $\varepsilon\left(\tau^{(+, n)}\right)=0$ and $\varepsilon\left(\tau^{(+, n)}\right)=1$

Define the probability mass function $\vec{\pi}(t ; \cdot, \cdot)$ with $i$-entry

$$
\pi_{i}\left(t ; n_{0}, n_{1}\right)=\mathbb{P}\left\{N_{0}(t)=n_{0}, N_{1}(t)=n_{1} \mid \varepsilon(0)=i\right\}, \quad n_{0}, n_{1} \geq 0, i \in\{0,1\} .
$$

First, note that with $n_{0}=n_{1}=0$ we have the differential equation

$$
\frac{\mathrm{d} \vec{\pi}(t ; 0,0)}{\mathrm{d} t}=(\mathscr{M}-\Lambda) \vec{\pi}(t ; 0,0)
$$

with the initial condition $\vec{\pi}(0 ; 0,0)=\mathbf{1}$. Here $\Lambda=\operatorname{diag}\left(\lambda_{0}, \lambda_{1}\right)$ and $\mathbf{1}=(1,1)^{\prime}$. The solution is

$$
\vec{\pi}(t ; 0,0)=\mathrm{e}^{(\mathscr{M}-\Lambda) t} \mathbf{1},
$$

see Fischer and Meier-Hellstern (1993, (22)).

Then, conditioning on the first switching we obtain the set of equations

$$
\begin{array}{ll}
\pi_{0}\left(t ; 0, n_{1}\right)=\int_{0}^{t} \mu_{0} \mathrm{e}^{-\mu_{0} u-\lambda_{0} u} \pi_{1}\left(t-u ; 0, n_{1}\right) \mathrm{d} u, & n_{1} \geq 0, \\
\pi_{1}\left(t ; n_{0}, 0\right)=\int_{0}^{t} \mu_{1} \mathrm{e}^{-\mu_{1} u-\lambda_{1} u} \pi_{0}\left(t-u ; n_{0}, 0\right) \mathrm{d} u, & n_{0} \geq 0,
\end{array}
$$

and, moreover

$$
\begin{aligned}
& \pi_{0}\left(t ; n_{0}, n_{1}\right)=\int_{0}^{t} \lambda_{0} \mathrm{e}^{-\lambda_{0} u-\mu_{0} u} \pi_{0}\left(t-u ; n_{0}-1, n_{1}\right) \mathrm{d} u+\int_{0}^{t} \mu_{0} \mathrm{e}^{-\lambda_{0} u-\mu_{0} u} \pi_{1}\left(t-u ; n_{0}, n_{1}\right) \mathrm{d} u, \quad n_{0} \geq 1, \\
& \pi_{1}\left(t ; n_{0}, n_{1}\right)=\int_{0}^{t} \lambda_{1} \mathrm{e}^{-\lambda_{1} u-\mu_{1} u} \pi_{1}\left(t-u ; n_{0}, n_{1}-1\right) \mathrm{d} u+\int_{0}^{t} \mu_{1} \mathrm{e}^{-\lambda_{1} u-\mu_{1} u} \pi_{0}\left(t-u ; n_{0}, n_{1}\right) \mathrm{d} u, \quad n_{1} \geq 1 .
\end{aligned}
$$


Eqs. (4.3)-(4.6) can be rewritten in the differential form

$$
\frac{\mathrm{d} \vec{\pi}\left(t ; n_{0}, n_{1}\right)}{\mathrm{d} t}=(\mathscr{M}-\Lambda) \vec{\pi}\left(t ; n_{0}, n_{1}\right)+\overrightarrow{\mathrm{g}}\left(t ; n_{0}, n_{1}\right),
$$

where $\vec{g}\left(t ; n_{0}, n_{1}\right)=\left(\lambda_{0} \pi_{0}\left(t ; n_{0}-1, n_{1}\right), \lambda_{1} \pi_{1}\left(t ; n_{0}, n_{1}-1\right)\right)^{\prime}$, presuming that $g_{i}\left(t ; n_{0}, n_{1}\right)=0$, if $n_{0}<0$ or $n_{1}<0$.

Therefore,

$$
\vec{\pi}\left(t ; n_{0}, n_{1}\right)=\int_{0}^{t} \mathrm{e}^{(\mathscr{M}-\Lambda)(t-u)} \overrightarrow{\mathrm{g}}\left(u ; n_{0}, n_{1}\right) \mathrm{d} u .
$$

Equations in (4.8) can be successively solved, using (4.2).

The solution can be expressed by using the spending time distributions.

Let $\mathscr{T}_{i}(t)=\int_{0}^{t} \mathbb{1}_{\left\{\varepsilon_{i}(u)=0\right\}} \mathrm{d} u, i \in\{0,1\}$, be the time spent by $\varepsilon_{i}(\cdot)$ in $(0, t)$ at the state 0 starting at the state $i$. The distribution of $\mathscr{T}_{i}(t)$ is well-known. The density functions of $\mathscr{T}_{i}(t)$ are given by

$$
\begin{aligned}
\psi_{0}(s, t)= & \mathrm{e}^{-\mu_{0} t} \delta(s-t)+\mathrm{e}^{-\mu_{0} s-\mu_{1}(t-s)}\left[\mu_{0} I_{0}\left(2 \sqrt{\mu_{0} \mu_{1} s(t-s)}\right)\right. \\
& \left.+\sqrt{\mu_{0} \mu_{1}} \sqrt{\frac{s}{t-s}} I_{1}\left(2 \sqrt{\mu_{0} \mu_{1} s(t-s)}\right)\right] \mathbb{1}_{\{0 \leq s \leq t\}}
\end{aligned}
$$

and

$$
\psi_{1}(s, t)=\mathrm{e}^{-\mu_{1} t} \delta(s)+\mathrm{e}^{-\mu_{0} s-\mu_{1}(t-s)}\left[\mu_{1} I_{0}\left(2 \sqrt{\mu_{0} \mu_{1} s(t-s)}\right)+\sqrt{\mu_{0} \mu_{1}} \sqrt{\frac{t-s}{s}} I_{1}\left(2 \sqrt{\mu_{0} \mu_{1} s(t-s)}\right)\right] \mathbb{1}_{\{0 \leq s \leq t\}},
$$

where $I_{0}$ and $I_{1}$ are the modified Bessel functions (Ratanov, 2010, (2.21)-(2.22)). See also Di Crescenzo and Zacks (2015), where sojourn times $\mathscr{T}_{i}(t)$ have been analysed in detail.

Therefore

$$
\begin{aligned}
& \pi_{0}\left(t ; n_{0}, n_{1}\right)=\frac{\lambda_{0}^{n_{0}} \lambda_{1}^{n_{1}}}{n_{0} ! n_{1} !} \mathrm{e}^{-\lambda_{1} t} \int_{0}^{t} s^{n_{0}}(t-s)^{n_{1}} \mathrm{e}^{-\left(\lambda_{0}-\lambda_{1}\right) s} \psi_{0}(s, t) \mathrm{d} s \\
& \pi_{1}\left(t ; n_{0}, n_{1}\right)=\frac{\lambda_{0}^{n_{0}} \lambda_{1}^{n_{1}}}{n_{0} ! n_{1} !} \mathrm{e}^{-\lambda_{1} t} \int_{0}^{t} s^{n_{0}}(t-s)^{n_{1}} \mathrm{e}^{-\left(\lambda_{0}-\lambda_{1}\right) s} \psi_{1}(s, t) \mathrm{d} s .
\end{aligned}
$$

Here $\psi_{0}(s, t)$ and $\psi_{1}(s, t)$ are the density functions of $\mathscr{T}_{0}(t)$ and $\mathscr{T}_{1}(t)$ respectively, see (4.9)-(4.10).

Note that from (4.11)-(4.12) it follows:

$$
\begin{aligned}
& \pi_{0}(t ; 0,0)=\mathrm{e}^{-\left(\lambda_{0}+\mu_{0}\right) t}+\int_{0}^{t} \bar{\psi}_{0}(s, t) \mathrm{e}^{-\lambda_{0} s-\lambda_{1}(t-s)} \mathrm{d} s, \\
& \pi_{1}(t ; 0,0)=\mathrm{e}^{-\left(\lambda_{1}+\mu_{1}\right) t}+\int_{0}^{t} \bar{\psi}_{1}(s, t) \mathrm{e}^{-\lambda_{0} s-\lambda_{1}(t-s)} \mathrm{d} s,
\end{aligned}
$$

where $\bar{\psi}_{0}$ and $\bar{\psi}_{1}$ are the regular parts of $\psi_{0}(s, t)$ and $\psi_{1}(s, t)$.

Consider two independent sequences of i.i.d. random variables $\left\{X_{0}^{(n)}\right\},\left\{X_{1}^{(n)}\right\}, n \geq 1$, with distribution functions $G_{0}$ and $G_{1}$ respectively. Let $J^{M}(t), t \geq 0$, be the compound process summing up the jumps alternatively from the sets $\left\{X_{0}^{(n)}\right\}_{n \geq 1}$ and $\left\{X_{1}^{(n)}\right\}_{n \geq 1}$,

$$
J^{M}(t)=\sum_{n=1}^{N(t)} X_{\varepsilon\left(\tau^{(+, n)}\right.}^{(n)} .
$$

The distribution of $J^{M}(t)$ is determined as follows.

Proposition 4.1. Under the given initial state $\varepsilon(0)=i$ the density function of $J^{M}(t)$ is

$$
p_{i}(x, t)=\pi_{i}(t ; 0,0) \delta(x)+\sum_{\substack{n_{0}, n_{1} \geq 0 \\ n_{0}+n_{1}>0}} \pi_{i}\left(t ; n_{0}, n_{1}\right)\left[f_{0}\left(\cdot ; n_{0}\right) * f_{1}\left(\cdot ; n_{1}\right)\right](x), \quad i \in\{0,1\} .
$$

Here $f_{0}\left(\cdot ; n_{0}\right), f_{1}\left(\cdot ; n_{1}\right)$ are the density functions of the sums $X_{0}^{\left(+, n_{0}\right)}, X_{1}^{\left(+, n_{1}\right)}$ respectively, and the probability mass functions $\pi_{i}\left(t ; n_{0}, n_{1}\right), n_{0}, n_{1} \geq 0$, are determined by (4.11)-(4.14). 
The distribution of $J^{M}(t)$ has an atom at 0 of size $\pi_{i}(t ; 0,0)$, (4.13)-(4.14), cf. Xu et al. (2015).

If jumps are positive and exponentially distributed (see Proposition 2.1), then $f_{k}(\cdot ; n)=f\left(\cdot ; n ; a_{k}\right), k \in\{0,1\}, n \geq 1$, are the Erlang-n densities, see (2.2). Therefore for $n_{0}, n_{1} \geq 1$

$$
\left[f_{0}\left(\cdot ; n_{0}\right) * f_{1}\left(\cdot ; n_{1}\right)\right](x)=\int_{0}^{x} \frac{a_{0}^{n_{0}} y^{n_{0}-1}}{\left(n_{0}-1\right) !} \mathrm{e}^{-a_{0} y} \frac{a_{1}^{n_{1}}(x-y)^{n_{1}-1}}{\left(n_{1}-1\right) !} \mathrm{e}^{-a_{1}(x-y)} \mathrm{d} y \mathbb{1}_{\{x>0\}} .
$$

Moreover, $f_{k}(x ; 0)=\delta(x)$. Hence for $n_{0}, n_{1} \geq 0, n_{0}+n_{1}>0$,

$$
\left[f_{0}\left(\cdot ; n_{0}\right) * f_{1}\left(\cdot ; n_{1}\right)\right](x)=\frac{a_{0}^{n_{0}} a_{1}^{n_{1}}}{\left(n_{0}+n_{1}-1\right) !} x^{n_{0}+n_{1}-1} \mathrm{e}^{-a_{1} x} \Phi\left(n_{0} ; n_{0}+n_{1} ;\left(a_{1}-a_{0}\right) x\right) \mathbb{1}_{\{x>0\}},
$$

see Gradshteyn and Ryzhik (1980, (3.383.2)).

In the case of the alternating signs of jumps (Proposition 2.2) we have

$$
\left[f_{0}\left(\cdot ; n_{0}\right) * f_{1}\left(\cdot ; n_{1}\right)\right](x)=a_{0}^{n_{0}} a_{1}^{n_{1}} \theta(x) \begin{cases}\frac{x^{n_{1}-1}}{a^{n_{0}}\left(n_{0}-1\right) !} \sum_{m=0}^{n_{1}-1} \frac{\left(n_{0}+m-1\right) !}{m !\left(n_{1}-m-1\right) !(a x)^{m}}, & \text { if } x>0, \\ \frac{|x|^{n_{0}-1}}{a^{n_{1}}\left(n_{1}-1\right) !} \sum_{m=0}^{n_{0}-1} \frac{\left(n_{1}+m-1\right) !}{m !\left(n_{0}-m-1\right) !(a x)^{m}}, & \text { if } x<0,\end{cases}
$$

for $n_{0}, n_{1} \geq 1$, see Gradshteyn and Ryzhik (1980, (3.381.4)). Moreover, $\left[f_{0}(\cdot ; 0) * f_{1}\left(\cdot ; n_{1}\right)\right](x)=f\left(x ; n_{1} ; a_{1}\right)$ and $\left[f_{0}\left(\cdot ; n_{0}\right) * f_{1}(\cdot ; 0)\right](x)=f\left(-x ; n_{0} ; a_{0}\right)$.

Eqs. (4.16)-(4.19) provide the explicit form of the distribution of the Markov-modulated compound Poisson process.

Remark 4.1. If one of the arrival rates is zero, such that $\lambda_{0}=0$ or $\lambda_{1}=0$, then the Markov-modulated Poisson process $N$ is called the interrupted Poisson process, which is equivalent to a hyper-exponential renewal process, Kuczura (1973) and Fischer and Meier-Hellstern (1993). In this case the times between switchings are distributed with the density function,

$$
A(t)=p \sigma_{1} \mathrm{e}^{-\sigma_{1} t}+(1-p) \sigma_{2} \mathrm{e}^{-\sigma_{2} t}, \quad t>0,
$$

where $p=\frac{\lambda-\sigma_{2}}{\sigma_{1}-\sigma_{2}}$ and

$$
\begin{aligned}
& \sigma_{1}=\frac{1}{2}\left[\lambda+\mu_{0}+\mu_{1}+\sqrt{\left(\lambda+\mu_{0}+\mu_{1}\right)^{2}-4 \lambda \mu_{1}}\right], \\
& \sigma_{2}=\frac{1}{2}\left[\lambda+\mu_{0}+\mu_{1}-\sqrt{\left(\lambda+\mu_{0}+\mu_{1}\right)^{2}-4 \lambda \mu_{1}}\right] .
\end{aligned}
$$

\section{Acknowledgement}

The author is grateful to the anonymous referee for careful reading and useful comments.

\section{References}

Di Crescenzo, A., 2001. On random motion with velocities alternating at Erlang-distributed random times. Adv. Appl. Probab. 33, 690-701.

Di Crescenzo, A., Martinucci, B., Zacks, S., 2015. Compound Poisson process with Poisson subordinator. J. Appl. Probab. 52 (2), $360-374$.

Di Crescenzo, A., Zacks, S., 2015. Probability law and flow function of Brownian motion driven by a generalized telegraph process. Methodol. Comput. Appl. Probab. 17 (3), 761-780. http://dx.doi.org/10.1007/s11009-013-9392-1.

Fischer, W., Meier-Hellstern, K., 1993. The Markov-modulated Poisson process (MMPP) cookbook. Perform. Eval. 18, 149-171.

Gradshteyn, I.S., Ryzhik, I.M., 1980. Tables of Integrals, Series and Products. Academic Press, NY.

Kolesnik, A.D., Ratanov, N., 2013. Telegraph Processes and Option Pricing. Springer, Heidelberg.

Kotz, S., Kozubowski, T.J., Podgórski, K., 2001. The Laplace Distribution and Generalizations. A Revisit with Applications to Communications, Economics, Engineering, and Finance. Springer.

Krall, H.L., Frink, O., 1948. A new class of orthogonal polynomials: the Bessel polynomials. Trans. Amer. Math. Soc. 65 (1), $100-115$

Kuczura, A., 1973. The interrupted Poisson process as an overflow process. Bell Syst. Tech. J. 52 (3), 437-448.

López, O., Ratanov, N., 2012. Option pricing driven by a telegraph process with random jumps. J. Appl. Probab. 49 (3), 838-849.

Ratanov, N., 2007. A jump telegraph model for option pricing. Quant. Finance 7, 575-583.

Ratanov, N., 2010. Option pricing model based on a Markov-modulated diffusion with jumps. Braz. J. Probab. Stat. 24, 413-431.

Ratanov, N., 2013. Damped jump-telegraph processes. Statist. Probab. Lett. 83, 2282-2290.

Ratanov, N., 2014. Double telegraph processes and complete market models. Stoch. Anal. Appl. 32, 555-574.

Ratanov, N., 2015. Telegraph processes with random jumps and complete market models. Methodol. Comput. Appl. Probab. 29 (3), 677-695. http://dx.doi. org/10.1007/s11009-013-9388-X

Ross, S.M., 2007. Introduction to Probability Models, ninth ed. Elsevier.

Saboor, A., Provost, S.B., Ahmad, M., 2015. A confluent hypergeometric generalized inverse Gaussian distribution. Pakistan J. Statist. 31 (1), $21-32$.

Xu, Y., De, S.K., Zacks, S., 2015. Exact distribution of intermittently changing positive and negative compound Poisson process driven by an alternating renewal process and related functions. Probab. Engrg. Inform. Sci. 29 (3), 385-397. 